\title{
Ethnologies
}

\section{Divination, pratiques de guérison et traditions islamiques parmi des femmes d'origine ouest-africaine à Montréal}

\section{Diahara Traoré}

Volume 37, numéro 1, 2015

URI : https://id.erudit.org/iderudit/1039661ar

DOI : https://doi.org/10.7202/1039661ar

Aller au sommaire du numéro

\section{Éditeur(s)}

Association Canadienne d'Ethnologie et de Folklore

ISSN

1481-5974 (imprimé)

1708-0401 (numérique)

Découvrir la revue

Citer cet article

Traoré, D. (2015). Divination, pratiques de guérison et traditions islamiques parmi des femmes d'origine ouest-africaine à Montréal. Ethnologies, 37(1), 175-192. https://doi.org/10.7202/1039661ar
Résumé de l'article

À partir d'une enquête ethnographique, cet article examine des pratiques de divination et de guérison parmi des immigrantes musulmanes d'origine ouest-africaine à Montréal, montrant ainsi la dynamique dialogique entre ces pratiques et la construction du religieux en contexte migratoire. En utilisant un cadre conceptuel inspiré de la théorie de Talal Asad sur l'anthropologie de l'islam, l'article présente une description de pratiques divinatoires observées entre 2008 et 2010, ainsi que le rapport que les femmes qui y participent entretiennent avec celles-ci. En particulier, l'auteure décrit des séances de divination par les cauris, ainsi que les rituels et pratiques de protection qui en découlent parmi les femmes participantes. L'auteure présente également des pratiques de guérison telles que décrites lors d'entretiens avec 24 femmes ouest-africaines provenant du Niger, du Mali, de la Guinée et du Sénégal. Dans le contexte d'une pluralité religieuse croissante dans la région de Montréal, cette étude démontre que, même à l'intérieur d'une tradition religieuse établie (l'islam), diverses traditions sont négociées et (re)construites en contexte migratoire. 


\title{
DIVINATION, PRATIQUES DE GUÉRISON ET TRADITIONS ISLAMIQUES PARMI DES FEMMES D'ORIGINE OUEST- AFRICAINE À MONTRÉAL
}

\author{
Diahara Traoré \\ Université de Montréal
}

\section{Introduction}

Talal Asad (1986) dans son «idée d'une anthropologie de l'islam» remet en question l'approche anthropologique selon laquelle l'islam constitue une structure sociale commune à toutes les sociétés musulmanes. Il tente de produire un cadre d'analyse qui tient compte de la diversité et de l'hétérogénéité sans pour autant renier les traditions communes qui en forment la base. Il s'agit donc ici d'un projet d'établir une définition de l'islam en tant que tradition discursive ayant des liens variés et divers avec l'individu moral, la gouvernance et l'organisation sociale, ainsi que la production du savoir. À cet effet, une pratique est islamique à partir du moment où celle-ci est autorisée par les traditions discursives de l'islam et transmise par des musulmans, qu'ils soient des savants, des dirigeants religieux ou des parents peu instruits (Asad 1986:14). Cette définition de l'islam, centrée sur le discours, permet d'accommoder les différences dans la pratique ainsi que la diversité qui existe à travers les sociétés musulmanes tout en maintenant un fil conducteur commun issu de l'héritage des traditions. Cet article s'inscrit dans ce cadre conceptuel en explorant la production du savoir islamique par des femmes musulmanes d'origine ouest-africaine sous trois aspects. Les connaissances que les gens ont à propos des rites et des croyances, constituent une dimension importante de l'étude du religieux. Lorsqu'il s'agit de pratiques religieuses, le savoir accompagnant ces pratiques consiste dans l'exégèse, les explications que donnent les pratiquants quant à l'histoire de la pratique religieuse en question, son sens, ses finalités. Ces connaissances peuvent soit être partagées par l'ensemble des musulmans, soit être le monopole de spécialistes. Cet article se base sur des données 
recueillies entre 2008 et 2010 lors d'un terrain ethnographique mené au sein de communautés ouest-africaines vivant dans la région de Montréal ainsi que les banlieues environnantes. Dans le cadre de ce terrain, 24 femmes ont été interviewées, et l'auteure a effectué de l'observation participante d'activités sociales et religieuses, ainsi que dans les domiciles des femmes. Les femmes, âgées entre 25 et 55 ans, sont originaires du Mali, du Niger, de la Guinée, et du Sénégal, et sont de confession musulmane. Les descriptions présentées dans cet article sont une combinaison d'entretiens et de notes de terrain. Cet article examine certaines pratiques courantes, bien que moins connues, parmi certaines femmes ouest-africaines musulmanes, dans le but d'articuler justement la complexité de la dynamique dialogique qu'elles entretiennent avec le savoir islamique. L'article décrit ensuite des pratiques de divination, et de guérison observées lors d'un terrain ethnographique, ainsi que des pratiques qualifiées de «magiques ». Finalement, l'article décrit des pratiques de guérison associées au savoir islamique parmi les femmes étudiées.

\section{Les musulmans ouest-africains à Montréal}

Les femmes rencontrées lors de cette étude étaient toutes originaires de pays de l'Afrique de l'Ouest francophone et résidaient toutes au Québec depuis un minimum de cinq ans. Au Québec, l'immigration ouest-africaine est beaucoup plus récente qu'en France, probablement en raison de la politique très restrictive de l'immigration au Canada qui refusait le droit d'immigrer sur la base de critères ethniques. La Loi sur l'immigration est abolie en 1962 (CIC 2000). L'immigration ouest-africaine débute donc dans les années 1960 avec une première vague d'étudiants africains sur des visas d'étudiant dans le cadre d'échange du Commonwealth et de la Francophonie (LeBlanc 2002). Dans les années 1980, elle augmente considérablement en partie à cause du durcissement des politiques d'immigration en France, ainsi que dans les autres pays d'Europe de l'Ouest, mais surtout à cause du contexte linguistique francophone et de la nouvelle politique d'immigration du Québec. Les immigrants s'installent principalement dans les grands centres urbains et en particulier à Montréal qui continue de regrouper 90 \% des nouveaux arrivants au Québec (CIC 2007). Au Québec, entre 1997 et 2006, le nombre d'immigrants en provenance de l'Afrique de l'Ouest était d'environ 7000 personnes (MICC Québec 2008); en 2008, 5000 Ouest-Africains résidaient à Montréal. Avec la diversité religieuse accrue, l'islam est sorti de la marginalité depuis les années 1980. Durant les deux dernières décennies, le nombre de musulmans au Canada est 
passé de 100000 à 600 000. Au Québec, $95 \%$ des musulmans vivent dans la région métropolitaine de Montréal. À Montréal, on comptait environ 200000 musulmans et une soixantaine de lieux de cultes il y a 10 ans, incluant les mosquées et les salles de prières (Daher 2003). Aujourd'hui, on peut estimer que le nombre de lieux de culte islamiques a largement augmenté. Contrairement aux pays d'Europe où les musulmans sont majoritairement originaires de la même région, tels les Turcs en Allemagne ou les Maghrébins en France, les musulmans du Québec sont d'origines géographiques et ethniques diverses et aucun groupe n'est dominant. Par conséquent, la population musulmane est très hétérogène, tant dans sa composition ethnoculturelle et linguistique que nationale. Au niveau des statuts socioéconomiques et professionnels, il y a également une grande diversité. Les orientations religieuses et politiques varient également : politisés ou non, sunnites ou chiites, pratiquants ou non pratiquants (Daher 2003). Les musulmans de Montréal constituent donc un groupe pluriel, avec des rituels et des pratiques sociales variés (Fortin, LeBlanc et LeGall 2008). Les groupes maghrébins et du Moyen-Orient demeurent largement majoritaires à ce jour. Les lieux de cultes islamiques sont situés dans différents quartiers de l'île de Montréal, surtout là où l'on observe des concentrations de résidents musulmans : à Ville-Saint-Laurent, Côte-desNeiges, Notre-Dame-de-Grâce, Parc-Extension, Pierrefonds, St-Léonard, St-Michel et Montréal-Nord. (Gagnon et Germain 2002). Pour les OuestAfricains musulmans, l'immigration pose la question de la pratique et de l'identité religieuse au Québec, surtout depuis le tournant du 21e siècle et après les attaques terroristes du 11 septembre 2001. Comme le souligne Mahmood Mamdani (2002), le 11 septembre 2001 a mis les musulmans au centre d'un débat culturel qu'il qualifie de «culture talk». Selon lui, les musulmans sont désormais très conscients de l'image des musulmans qui circule dans les médias, et de ce qui est fait la différence entre les «bons » musulmans et les « mauvais » musulmans (Mamdani 2002).

\section{La divination : une consultation avec une lanceuse de cauris}

Benjamin Soares (2005) mentionne des pratiques féminines de l'islam en Afrique de l'Ouest, consistant principalement de pratiques divinatoires pré- et non-islamiques. Ce « savoir des femmes » n'occupe que deux pages et demie chez Soares (2005: 145-148) et est très vite discrédité par ses informateurs, des hommes maliens :

Many men in Nioro were quick to note that Muslim women's secrets were not as licit as some of their own secrets, which they claimed were 
tied directly to Islam and its correct interpretation. They argued that because women have much less knowledge of Islam and its central text, the Quran, than men, their practices were necessarily more questionable, possibly even unlawful (Soares : 145).

Ce sont précisément ces «pratiques douteuses et possiblement même illégitimes » qui nous intéressent ici, notamment en examinant la divination par les cauris, à laquelle la majorité des femmes rencontrées dans le cadre de notre terrain ethnographique. Le terme divination fait référence aux techniques mises en œuvre pour interpréter des évènements passés, présents ou futurs. Les institutions divinatoires existent dans la plupart des sociétés. Pour plusieurs peuples africains, le "véritable » savoir est caché, secret, et accessible uniquement à certaines personnes capables de l'utiliser de manière appropriée (Peek 1991). Les techniques divinatoires sont diverses et coexistent souvent au sein d'une même société. Les pratiques divinatoires en Afrique de l'Ouest ont toujours joué un rôle important dans la conduite des affaires familiales, communautaires et même politiques. Dans le cadre de nos observations, nous nous sommes limités à la pratique divinatoire des cauris. Les cauris sont des coquillages qui servaient communément en Afrique de l'Ouest précoloniale de monnaie d'échange. Les cauris avec lesquels est pratiquée la divination sont généralement plus petits que ceux utilisés comme monnaie. Le lancement de cauris est un procédé divinatoire très répandu en Afrique subsaharienne musulmane (Regourd 2000), surtout dans les quatre pays d'origine des femmes de l'étude, comme le souligne une femme sénégalaise de l'étude :

Au Sénégal, j’ai déjà, je suis allée voir une femme qui faisait les cauris, juste pour le fun. C'est arrivé une ou deux fois qu'on est allé avec des amis et y avait une femme qui fait les cauris et qui l'a fait. C'est quelque chose qui est très courant chez nous. (Dada, 8 mai 2009, Montréal) ${ }^{1}$

C'est une méthode de divination utilisée à des fins diverses, telles que " lire les auspices sous lesquelles se déroulera une manifestation, ou pour savoir si un sacrifice a été accepté par les divinités » (Dramé 1986). On l'utilise pour connaître l'issue d'un événement à venir, l'avenir immédiat ou à long terme d'un individu, ou pour déterminer le type de sacrifices à accomplir pour obtenir une issue favorable dans une épreuve. La volonté des divinités ou esprits consultés est transmise au devin à travers les cauris

1. Les noms utilisés sont des pseudonymes afin de protéger l'anonymat des femmes étudiées. 
sous forme de code basé sur le positionnement des cauris dans un panier. Cette pratique est généralement transmise de mère en fille, ou d'une femme plus âgée à une plus jeune de la même famille. Selon certaines croyances, on peut également recevoir le don d'interprétation des cauris en songe, lorsque l'on se voit se faire offrir une poignée de cauris par quelqu'un. La divination par les cauris est en réalité un mode de communication entre les humains et les esprits ou divinités. Dans plusieurs cultes yorubas au Nigéria par exemple, cette pratique est utilisée pour connaître la volonté des dieux, ainsi que les mesures à prendre pour s'assurer leurs bonnes faveurs dans la vie courante (Bascom 1980). Bien que la divination soit reconnue comme relevant du domaine des hommes, la divination par les cauris demeure la plus pratiquée par les femmes (Ogdubesan 1969). La religion islamique apporte une nouvelle cosmologie et de nouvelles conceptions des pratiques divinatoires. On ne parle désormais plus de consulter les ancêtres ou les divinités locales, mais de consulter Dieu, tandis que d'autres pratiques sont remplacées par les aumônes, comme le montre une étude sur la divination et les croyances islamiques au Ghana (Kirby 1993).(Regourd 2000). La pratique de consultation deDieu par des songes par le biais de prières islamiques spéciales de consultation, al-istikhara, est reconnue comme acceptable par l'islam. Néanmoins, comme le constate Rosalind Shaw dans son étude sur la divination par songe chez les Temné, les croyances islamiques, plutôt que d'éradiquer les croyances locales, ne font que contribuer à la cosmologie, en se juxtaposant aux pratiques et conceptions existantes (Shaw 1992).

Une de nos observations nous a menés dans un appartement du quartier Saint-Laurent à Montréal, chez Madame Djénabou², pour accompagner deux femmes de mon étude, en septembre 2008 puis à nouveau en novembre 2010. Celle-ci, 42 ans, a été initiée au déchiffrage des cauris par sa tante maternelle, en Guinée. Musulmane pratiquante, elle exhorte ses clientes à faire leurs prières régulièrement.

Madame Djénabou offre pour la somme modique de 20 \$ une séance de consultation de l'avenir à l'aide de cauris. La plupart de ses clients sont des hommes et des femmes originaires de l'Afrique subsaharienne, incluant plusieurs femmes musulmanes ouest-africaines. En arrivant chez Madame Djénabou, il faut sonner à la porte de l'immeuble et s'identifier. Madame Djénabou n'ouvrira que si elle reconnaît le nom de la personne qui vient la consulter. Il faut, au préalable, avoir été référé par un de ses clients et avoir pris un rendez-vous. Ceci s'explique par le fait que la divination est

2. Il s'agit d'un pseudonyme. 
pratiquée dans le secret et l'anonymat des clients doit être préservé. Une fois dans son domicile, on est invité à s'assoir au salon en attendant Madame Djénabou. On s'y retrouve la plupart du temps seul, car Madame Djénabou planifie les rendez-vous en s'assurant que les clients ne se rencontrent pas chez elle. Son salon est décoré avec soin : des fauteuils confortables en cuir, des tables basses de verre, des plantes vertes, et des décorations diverses, telles que des tableaux ou des sculptures africaines. Une télévision à un écran plat de 37 pouces offre un éventail de chaînes internationales. Madame Djénabou émerge de sa chambre et, après les salutations d'usage, invite la cliente à la suivre à l'intérieur, à l'abri des oreilles indiscrètes. Les consultations ont lieu dans la chambre de ses enfants, dans l'espace entre le mur et le lit superposé de ceux-ci. Assise l'une en face de l'autre, Madame Djénabou et la cliente commence la consultation. Madame Djénabou se pose sur les genoux un panier plat et rond en rotin dans lequel se trouvent douze cauris. La cliente est invitée alors à déposer de l'argent symboliquement dans le panier afin d'inciter les cauris à parler. Une pièce d'un dollar suffit généralement. Madame Djénabou invite alors la cliente à prendre les cauris dans sa main et à leur dire à voix basse la raison de sa visite ou les questions qui la troublent. La séance de divination peut enfin débuter. Madame Djénabou ramasse les cauris dans sa main droite, puis les lance dans le panier. Nos notes de terrain offrent, à cet égard, une description de la séance :

Madame Djénabou ramasse les cauris avec sa main droite, tenant le panier de sa main gauche. Elle les jette ensuite dans le panier et déchiffre le positionnement de chaque coquillage, avant de secouer la tête d'un air dubitatif. S'ensuit qu'elle commence à me parler du sujet même que j'ai mentionné mentalement en tenant les cauris dans ma main. Lorsque les nouvelles sont mauvaises, Madame Djénabou m'invite à faire mes prières islamiques régulièrement « Il faut vraiment toujours prier. Tu sais que nous sommes des musulmans. Et même si la prière est difficile parfois, c'est cela qui nous protège de tous les maux ». Elle me dit ceci en dioula. Puis précise qu'il me faut verser de l'eau (dji bon) après chaque prière. Je fronce les sourcils. « Jeter de l'eau? » je lui demande. " Oui, tu ne connais pas ça?! Mais c'est très important de faire ça hein. " «C'est pour que les plans de tes ennemis contre toi n'aboutissent pas. » Elle m'explique que quand on vit dans un appartement cela peut se faire en versant de l'eau dans un pot à plante. Les bonnes nouvelles, elles, sont suivis d'une invocation divine que moi, la consultante, je dois faire en tenant dans ma main les cauris annonceurs de bonne nouvelle et en les maintenant contre mon front en disant «Que Dieu l'exauce » Allah koo kê. Elle me 
conseille ensuite de faire du sacrifice de lait à des petits enfants, et également $\mathrm{d}$ bonbons et de biscuits. Elle me dit aussi de donner des noix de colas en me précisant la couleur et le type de ces noix de cola à un musulman. Le don doit être fait à " quelqu'un qui prie " un musulman. (Notes de terrain, 8 septembre 2008, Saint-Laurent)

Par ailleurs, la prière rituelle islamique est affirmée comme une pratique nécessaire et complémentaire aux prescriptions communiquées par les cauris. Lorsque Nana, originaire du Sénégal et âgée de 27 ans vient consulter Madame Djénabou, la lanceuse de cauris lui révèle tout de suite la cause de sa visite. Après avoir lancé les cauris deux ou trois fois dans le panier en secouant la tête, dubitative, Madame Djénabou lui annonce que l'homme que Nana fréquente, souhaite l'épouser. Nana est inquiète. Malgré une relation amoureuse qui dure maintenant depuis six mois, Aziz, un sénégalais de 34 qui vit à Boston, ne fait pas mention de mariage. Les cauris annoncent une bonne nouvelle : Aziz l'aime et veut la demander en mariage. Madame Djénabou tend les cauris porteurs du message à Nana, et celle-ci les prend dans sa main, puis les porte à son front en disant : " Qu'Allah l'accomplisse ». La lanceuse de cauris réplique aussitôt « Amine ». La séance se poursuit.

La consultation révèle à Nana qu'il lui faut absolument faire ses cinq prières islamiques par jour, et surtout s'assurer de verser de l'eau après chaque prière. En interrogeant Madame Djénabou sur la pratique de verser de l'eau après chaque prière, nous apprenons que cette une pratique importante permet d'éloigner les malheurs et s'attirer une protection. Nana, par ailleurs, n'est elle-même pas surprise : «Au Sénégal, on m'avait déjà dit de faire ça, mais c'est vrai que j'avais arrêté (de verser l'eau). J'ai négligé, en fait ». Suite à nos questions, Madame Djénabou clarifie que normalement l'eau se verse sur le sol de terre, mais qu'étant à Montréal, on peut la verser dans un pot de plante. Nana reçoit également une liste de sacrifices à accomplir pour s'assurer une issue favorable : trois noix de cola ni blanches ni sombres, et du lait à donner à des petits enfants. Le sacrifice, ou saddaqa, est important dans la mesure où il permet d'éviter un malheur futur (Werbner 2015). Selon Claude Lévi-Strauss (1962), le rite sacrificiel a pour but de créer un rapport « entre deux termes polaires dont l'un est le sacrificateur et l'autre la divinité, et entre lesquels, au départ, il n'existe pas d'homologie, ni même de rapport d'aucune sorte » (Lévi-Strauss 1962:196). Le sacrifiant, c'est-à-dire le sujet qui recueille les bienfaits ou subit les effets du sacrifice acquière par le sacrifice un changement d'état, voire même une sorte de «transformation religieuse » de son statut (Hubert 
et Mauss 1968). Dans son étude sur le destin dans la société sénégalaise, Ibrahima Sow fait état de l'absence de rigidité et l'élasticité d'un destin auquel un autre peut être substitué par le biais de sacrifices : « Il semble qu'à un destin énoncé et prédit, il peut être substitué un autre destin qu'il faut auparavant satisfaire et réaliser pour que puisse s'accomplir la totalité du verdict destinal » (Sow 2004 : 51). En accomplissant le rituel du sacrifice, Nana pourra substituer un destin remplis d'embûches à un destin où l'accès au mariage est facilité. En établissant un rapport avec Dieu par le biais de son sacrifice, elle bénéficiera d'une transformation de son statut religieux qui lui attirera donc la faveur de Dieu, afin que Dieu puisse changer son destin et lui éviter le malheur ou l'obstacle prédit. Pour que le sacrifice puisse être bénéfique, il faut suivre des instructions précises concernant notamment le récipiendaire de l'offrande en question: une femme âgée « qui prie », ou des «petits » enfants, ou encore, un homme « qui prie ». L'expression « qui prie » fait référence ici à un musulman, une personne qui observent les rituels de l'islam et en particulier qui accomplit ses cinq prières par jour. Ceci peut être interpréter comme une volonté d'islamiser cette pratique et de l'inscrire dans le contexte de la zakaat islamique, qui implique des dons de charité envers les défavorisés de la communauté musulmane. D’ailleurs, une des femmes interrogées parle des sacrifices comme l'une des pratiques régulières de l'islam, avec la prière :

Nous comme on était des enfants, on faisait quand même notre mieux pour faire les cinq prières. C'est les vieilles qui ajoutaient les nafila ${ }^{3}$. Et puis aussi il faut faire des sacrifices dans la vie. Par exemple tu peux donner du lait aux enfants ou bien donner de l'argent aux jumelles ou aux jumeaux ou bien d'acheter du cola de donner à la mosquée ou à une vieille personne qui prie. Donc des sacrifices comme ça ou de l'aumône a des gens qui sont pauvres. Si tu as des voisines qui sont pauvres à côté, tu peux leur donner à manger. (...) Je suis pratiquante. Je fais mes prières et je fais les sacrifices qu'il faut si j'ai les moyens, c'est ça. (Counda, 21 janvier 2009, LaSalle)

Une autre femme rencontrée, responsable d'une garderie en milieu familial, en tentant d'expliquer ses pratiques islamiques dans le contexte montréalais, mentionne que :

Moi je me dis tous les jours je donne des sacrifices parce que chez moi ici c'est toujours plein, les neveux et nièces qui étudient à l'université, le weekend ils sont ici. Et vu que j'ai une garderie, je donne toujours du lait aux enfants. Et normalement les parents doivent amener le

3. Prières islamiques supplémentaires optionnelles. 
lait eux-mêmes, mais j'ai dit à mon assistante que je vais pas exiger ça, ça je peux l'offrir. Ce sont des petits trucs comme ça qui sont des sacrifices pour moi. (Nafi, 1er octobre 2009, Outremont)

Selon la pratique usuelle, pendant que l'on donne l'offrande au récipiendaire, on s'assure de demander des prières d'intercession. Le récipiendaire, par ailleurs, fait des bénédictions en invoquant le nom de Dieu, tandis que le donateur les ponctue de « amine » sonores. Dans le cas de Nana, elle se rend dans une épicerie africaine de Montréal pour trouver des noix de colas appropriées. Il est très important de suivre les instructions de la lanceuse de cauris à la lettre. Ne pas effectuer le sacrifice correctement peut avoir des conséquences sérieuses, allant jusqu'à la substitution de l'ancien destin d'embûche par un destin affranchi d'obstacle peut ne pas avoir lieu.

Ces pratiques divinatoires, ainsi que les sacrifices mentionnés plus haut, font partie d'un ensemble de croyances en un monde invisible, monde qui peut tout autant bénir que nuire au bonheur. Les djinns ou les jnoun font partie des croyances inscrites dans le dogme islamique au même titre que les anges, les livres saints, le paradis et la résurrection. Ces êtres invisibles sont classés en deux catégories : des bénéfiques et des maléfiques (Burge 2014, Khan 2006). Ils sont partout, invisibles tout en étant proches de nous, actifs plutôt la nuit que le jour, pouvant se manifester sous une forme humaine ou animale, capables de se marier avec les humains, de les séduire (Rothenberg 2006), et de punir celui ou celle qui les dérange, les blesse, les offense ou simplement empiète sur leurs domaines ou leurs lieux de résidence (Islam et Campbell 2014). Certains djinns sont musulmans, d'autres sont chrétiens, juifs ou païens. Leur univers est complexe et intimidant pour le non-initié (le client du devin) et toute communication avec lui, en dehors de tout rite ou de toute protection, est dangereuse et peut exposer à plusieurs maux et malheurs, une réalité qui exige de se protéger.

Deux ans après sa consultation avec Madame Djénabou, nous retrouvons Nana qui revient d'un long séjour au Sénégal en 2010. Elle a un nouveau prétendant pour le mariage, mais celui-ci ne semble pas se décider pour une date de mariage. Nana s'explique l'hésitation de cet homme : «Le problème c'est qu'il ne prie pas. Si au moins il priait, tous ces trucs mystiques que les gens font pour ne pas qu'il se marie... tout ça n'allait pas marcher. »(Nana, 12 novembre 2010, Verdun) C'est d'ailleurs un marabout musulman qui a confirmé à Nana la cause mystique de l'indécision de son prétendant : des ennemis auraient fait « un travail »-jeté un sort - afin de le bloquer dans ses entreprises. Ce marabout, selon la description de Nana, a procédé à des prières islamiques spéciales, 
nommée al-istikhar (terme arabe pour questionnement ou consultation). Après avoir fait ces prières, le marabout a eu accès à la réponse aux questions par le biais de songes (Nana, 12 novembre 2010). Cette pratique est bien décrite par Ibrahima Sow $(1977,2006)$ :

Cette technique se pratique à la demande d'un consultant qui, lorsqu'il a des ennuis personnels ou qu'il souhaite qu'un projet aboutisse, va exposer minutieusement son cas au marabout (praticien musulman). Les projets personnels vont-ils aboutir? Les ennuis vont-ils cesser? Telles sont, finalement, les questions dont on attend la réponse. Le consultant se retire; quant au " marabout ", il opère la nuit et donne ses réponses sous huitaine au plus tard. C'est parce qu'il y a des jours favorables pour pratiquer le listixaar. Les nuits du dimanche au lundi, et du jeudi au vendredi semblent être tout particulièrement indiquées. Il s'agit, pour le marabout, de se mettre dans des conditions telles qu'il se trouve dans un état de rêve éveillé durant lequel se déroule, « comme un film devant ses yeux », le cas du consultant. (Sow, $1977: 53$ ).

\section{Pratiques magiques et protection spirituelle}

En addition aux pratiques divinatoires, nous avons observé des pratiques que nous qualifions de magiques. En parlant de magie dans le contexte des femmes de ma recherche, nous faisons référence à l'usage de talismans, d'objets ou de rituels dans le but d'influencer le comportement d'autrui, de changer le cours des évènements ou de nuire à autrui. La magie consiste en savoirs, croyances et pratiques partagées, qui vont émerger par besoin d'agir sur des forces indéchiffrables et impersonnelles (mana), inhérentes à la nature ou à certaines personnes (Mauss et Hubert 1950). L'objectif est de manipuler des forces ou encore à les capter dans le but de s'en servir. Levi-Strauss (1958) observe que la magie que le chamane amérindien opère lors des accouchements compliqués agit en transformant le matériau mythique et symbolique, par le biais de transferts symboliques. Ce transfert symbolique est à la base de plusieurs pratiques, comme discuté ci-dessous.

Une femme de l'étude, Aminata, originaire du Mali et ayant des problèmes conjugaux m'a raconté qu'elle a recouru à cette méthode afin d'influencer le comportement de son mari. Elle a récité certains chapitres du Coran, notamment, le verset « Le Trône » dans le chapitre « La Vache » du Coran et a ensuite soufflé dans un verre de lait qu'elle a fait boire à son époux. Son époux, qui s'opposait à ce qu'elle se rende au Mali pour les vacances, a finalement donné son accord. Cette pratique rappelle 
celle des femmes algériennes dans l'étude de Marnia Lazreg (1994) sur les pratiques précoloniales mystiques des femmes. L'auteure met en garde contre une lecture des pratiques féminines de magie comme indication de leur impuissance face à leur condition (Lazreg 1994 : 113-114). Bien que Lazreg interprète ces pratiques comme n'étant que de simples activités de passe-temps, notre terrain ethnographique révèle que, loin d'être de simples passe-temps, ces pratiques sont des moyens de retrouver un contrôle sur un destin et des circonstances qui les dépassent.

Naturellement, la protection spirituelle contre des attaques mystiques liées à la sorcellerie constitue un aspect important de ces pratiques, qui sont prises très au sérieux par certaines femmes. Awa, 25 ans et originaire du Mali, s'était fait refuser plusieurs demandes de visas d'étude par la France avant son immigration au Canada. Un marabout local l'avait informée de la cause de ces embûches : une tante maternelle avait usé de pratiques occultes contre elle afin de lui fermer les portes des études et du travail. Awa avait reçu du marabout des concoctions à base de feuilles et qui contenaient l'encre utilisée pour écrire le chapitre Ya-Sin du Coran. Elle raconte qu'elle devait se frictionner tout le corps avec ces concoctions quotidiennement : « Heureusement que moi aussi je priais beaucoup. C'est là que j'ai vraiment commencé à faire mes cinq prières parce que j'ai vu que c'était grave. » (Awa, 28 mai 2009, Montréal)

Par la suite, Awa a obtenu un visa pour le Canada. Elle est toujours en contact téléphonique plus ou moins régulier avec le marabout qui l'a aidé à Bamako, et malgré la distance, continue de le consulter. De fait, la pratique des prières islamiques est interprétée comme l'un des remèdes aux attaques mystiques et de sorcellerie. Dans le cas de Awa, elle décide de faire ses prières régulièrement afin de renforcer l'effet des concoctions de son marabout. La prière assidue constitue donc un rempart contre les mauvais sorts et les complots d'ennemis divers, comme l'exprime cette autre femme :

Parce que, en islam il y a certaines sourates si tu lis ça, ça peut t'aider beaucoup. Par exemple, ma mère m'a dit le matin si tu te réveilles, tu prends un verre d'eau avant que tu te brosses, tu dis bismillahi et puis tout ça-là et puis tu bois et c'est bon pour ta santé. (Counda, 21 janvier 2009, LaSalle)

On est proche ici de la notion de « sécurité spirituelle » que décrit Adam Ashforth dans son ouvrage sur la sorcellerie et la violence en Afrique du Sud (2005). Incontestablement, le monde invisible étant plein de menaces avec des ennemis difficilement identifiables, le sentiment d'insécurité est 
omniprésent. Des problèmes de santé à répétition, ou encore des échecs successifs sont souvent perçus comme des attaques de parents ou d'amis jaloux. Comme l'explique, Mouminatou, une femme sénégalaise mouride, il peut arriver que certains problèmes de santé ne soient pas liés à des causes naturelles :

Bon comme je te dis, mon guide religieux Cheikh Ahmadou Bamba, il a écrit beaucoup de poèmes et à chaque poème sa spécificité. C'est à dire des choses qu'ils peuvent te permettre d'accomplir... Et... y en un poème qui permet vraiment de guérir certaines maladies. Si tu l'apprends, quand tu es malade tu les lis, ça peut être comme une prière qui permet de guérir ta maladie, ça, par la grâce de Dieu quand même... Mais je sais que ce poème-là a ce pouvoir-là. Mais moi particulièrement une fois que je suis malade c'est instantané, je vais voir un médecin, tu prends des médicaments. Mais en plus des médicaments je fais des prières à coté aussi. Surtout quand c'est une maladie qui dépasse un peu les choses que je comprends. (Mouminatou, 10 juin 2009, Montréal)

À cet effet, pour les femmes interrogées, les rituels de l'islam sont logés à la même enseigne que les pratiques de divination et les talismans. Notamment, les talismans sont souvent fabriqués avec des versets coraniques (Hamès 2007). Les versets coraniques sont écrits sur du papier ou sur une tablette de bois. L'encre est lavée avec de l'eau et le liquide recueilli est patiemment séché. La poudre obtenue est insérée dans une pochette de cuir, qui une fois recousue, est portée sur le corps comme ceinture ou bracelet.

Parmi les femmes d'origines malienne et sénégalaise, nous avons constaté la popularité d'un ouvrage en Afrique de l'Ouest qui est représentatif des pratiques et rituels couramment utilisés. L'ouvrage, Les Dimensions de l'islam (1996), par Cheikh El Hadji Ahmadou Tall, décrit les différents usages des 99 noms de Dieu, ainsi que le nombre de fois que chaque nom doit être récité afin d'influencer les évènements, obtenir une guérison, ou encore disposer une personne en faveur du requérant. L'auteur, un adepte de la Tidjaniyya, est reconnu en Afrique de l'Ouest comme un érudit de la numérologie islamique. Certains chiffres sont associés à des noms divins spécifiques. Plusieurs des femmes de cette étude possédaient une copie de cet ouvrage dans leur domicile et en ont fait référence pendant les conversations informelles ou les entretiens. Pour plusieurs, les pratiques de ces femmes donnent l'impression d'être difficilement dissociable de pratiques hétérogènes provenant d'autres traditions. C'est par exemple une impression partagée par Vincent Monteil 
(1964) qui fait le constat d'un islam qu'il qualifie d' « islam noir », un islam syncrétique et spécifique à l'Afrique de l'ouest. De même, René-Luc Moreau (1982) parle des «Africains musulmans » en des termes similaires, appuyant l'idée d'un islam typique de l'Afrique de l'Ouest qui se distingue par l'incorporation de pratiques issues de traditions locales. Pour Levtzion (1979), en Afrique de l'Ouest l'islamisation est un passage progressif qui s'opère de façon collective, impliquant un passage par le syncrétisme qui ne serait qu'une étape dans le processus de conversion, par lequel on passerait d'un islam nominal à une intégration complète à l'orthodoxie (Triaud 1974). Ces auteurs avaient une vision de l'islam en Afrique au Sud du Sahara comme étant plus syncrétique que l'islam ailleurs, ce qui n'est guère surprenant car leurs travaux datent de l'époque de l'aprèsindépendance. Dans la littérature qui pré-date les années 1990, l'idée de cet islam syncrétique est omniprésente dans la littérature (Monteil 1964; Moreau 1982; Levtzion 1979; Triaud 1974).

Selon nos constats, le cadre conceptuel Asadien qui conçoit l'islam comme un ensemble de traditions discursives offre un éclairage moins teinté par ces classifications binaires (islam syncrétique v. islam) et la notion d'une évolution progressive vers une pratique plus puriste de l'islam. Nous ne nous inscrivons pas dans la logique de distinguer un islam soit disant pur d'un islam syncrétique pour plusieurs raisons. Premièrement, dans quelque contexte social que ce soit, les pratiques religieuses ne peuvent être dîtes pures ou moins pures, car l'argument lié à l'authenticité ne peut être scientifique. Il s'agit plutôt d'un argument idéologique. Nous tenons particulièrement à me distinguer de cet argument dans la mesure où celuici qualifie les pratiques divinatoires ou magiques des femmes de pratiques syncrétiques ou de confusions entre culture et religion. Deuxièmement, l'argument mis de l'avant dans cet article s'inscrit dans un projet de mettre à jour des traditions subsédiaires. Par ailleurs, notre intention n'est pas de s'aligner sur les arguments ou théories de ces divers auteurs, mais de décrire les pratiques des femmes étudiées selon mes observations et leurs propres récits. D'évidence, les femmes étudiées ne se perçoivent pas comme étant moins musulmanes de par leurs pratiques, comme en témoignent leurs discours. Au contraire, selon elles, c'est justement en pratiquant l'islam que l'on se protège des dangers que la divination révèle.

Dans cette même optique de protection spirituelle, on s'abstient de révéler à autrui ses projets (mariage, promotion professionnelle, achat de propriété...) jusqu'à ce que les plans soient accomplis. Les femmes musulmanes d'origine ouest-africaine de l'étude qui ont été enceintes ont 
admis qu'elles n'annoncent pas leur grossesse publiquement. Ce n'est que lorsque l'arrondi du ventre est visible, que la nouvelle est implicitement annoncée. Incidemment, un proverbe bambara décrit une femme enceinte comme ayant un pied dans la vie et un pied dans la mort. Ashforth fait le même observation en Afrique du Sud, dans des circonstances quelques peu embarrassantes :

So when the neighbor inquired, "Where's Seipati? » I replied with the simple, stupid, truth: «In the hospital having her baby. » Almost before I could finish the sentence I was drawn up short by an alarmed flare from MaMfete. I knew then that I had committed more than a gaffe. I had been around long enough by then to know I should have known better. (...) The moments of birth and death, when persons are in the process of transition from one form of being to another, are particularly vulnerable to attack by malicious agents using invisible forces. Discretion is of the utmost importance at such times in order to secure the mother and child from hostile interference or to allow safe passage for the deceased into the afterlife. Discretion at times of childbirth is also more generally important to prevent the circulation of information that might give rise to jealousy. (Ashforth 2005:73)

La croyance en des menaces invisibles, mais bien réelles, informe les pratiques de certaines femmes de l'étude. De la même façon, une grande partie des femmes musulmanes interrogées ont mentionné des pratiques de guérison associées à des récitations de versets du Coran. En cela, il n'y a rien de nouveau surtout à la lumière des traditions prophétiques concernant les vertus guérisseuses de certains versets ou de certains noms de Dieu, qui datent du premier siècle de l'islam. Bien que toutes les femmes sans exception aient affirmé n'avoir aucune réserve face à l'utilisation de versets du Coran à des fins de guérison, cette dernière est pratiquée dans la discrétion. C'est ainsi que certaines femmes utilisent la récitation de certains versets coraniques, où dans le cas des mourides, de poèmes écrit par Ahmadou Bamba, le fondateur de la confrérie mouride. Par exemple, les propos de Mame Faye attestent de ce fait :

Mon guide religieux Cheikh Ahmadou Bamba, il a écrit beaucoup de poèmes et à chaque poème sa spécificité. C'est à dire des choses qu'ils peuvent te permettre d'accomplir... Et... y en un poème qui permet vraiment de guérir certaines maladies. Si tu l'apprends, quand tu es malade tu les lis, ça peut être comme une prière qui permet de guérir ta maladie, ça, par la grâce de Dieu quand même. (Mame Faye, 19 avril 2009, LaSalle) 
Ce type de pratiques était partagé à la majorité des femmes interrogées. Une autre femme explique qu'elle a hérité cette pratique de sa mère :

Moi, c'est systématique. Même quand je me sens pas bien, mal de tête, je récite des versets du Coran. Il m'est arrivé d'être malade et puis de réciter un verset de Coran sur un verre d'eau et d'aller me coucher et de le verser devant ma porte. Je ne sais pas si c'est considéré en tant que tel. Mais c'est quelque chose de courant que j'ai toujours fait. Par mon éducation familiale, c'est ma mère qui n'est pas musulmane de naissance qui me l'a montré quand je me réveille en pleine nuit et que je me sens lourde et pas bien, elle me dit tu récites des versets et tu fais deux rakat et ça va aller. (Dada, 8 mai 2009, Montréal)

\section{Conclusion}

Bien que ces pratiques propres aux femmes ne se limitent pas à des formes non légitimes - légitime ici faisant référence aux savoirs reconnus par les figures de l'autorité en islam - ces femmes imbriquent les formes légitimes aux formes non légitimes. Dans la pratique et dans la conception de certaines des femmes ouest-africaines étudiées, cette imbrication de traditions est constamment renégociée, comme en témoigne Madame Djénabou dans sa façon de qualifier l'islam, à savoir nécessaire et complémentaire aux pratiques de divinations. Il en découle une pluralité de discours, qui bien que s'opposant parfois, contribuent à définir l'islam. Dans la pratique et dans la conception de certaines des femmes ouest-africaines étudiées, cette imbrication de traditions est constamment renégociée, comme en témoigne Madame Djénabou dans sa façon de qualifier l'islam, comme complémentaire aux pratiques de divination. Construire un discours sur l'islam qui est adaptable, juxtaposable à des pratiques magiques, ou au contraire exclusif de ces dernières, devient un acte perpétuel de redéfinition de ce que constitue l'islam dans le contexte de la vie quotidienne. 


\section{Références}

Asad, Talal, 1986, The Idea of an Anthropology of Islam, Washington, DC : Georgetown University Press.

Ashforth, Adam, 2005, Witchcraft, violence, and democracy in South Africa.

Chicago: University of Chicago Press.

Bascom, William, 1980, Sixteen Cowries: Yoruba Divination from Africa to the New World. Bloomington: Indiana University Press.

Burge, Stephen, 2012, "Islam, Arabs, and the Intelligent World of the Jinn (Contemporary Issues in the Middle East Series), Amira El-Zein ». British Journal of Middle Eastern Studies, 39(3) : 427-429.

Citoyenneté et immigration Canada, 2007, Faits et chiffres 2007. Aperçu de l'immigration : résidents permanents. Ottawa: Gouvernement du Canada.

Citoyenneté et immigration Canada, 2000, Les artisans de notre patrimoine : la citoyenneté et l'immigration au Canada de 1900 à 1977. Ottawa : Gouvernement du Canada.

Daher, Ali, 2003, Les musulmans au Québec. Un document produit en version numérique par Jean-Marie Tremblay, Cégep de Chicoutimi. Bibliothèque Paul-Émile-Boulet, Université du Québec à Chicoutimi. Récupéré de http://classiques.uqac.ca/contemporains/daher_ali/ musulmans_au_quebec/musulmans_au_Quebec.pdf.

Dramé, Mallafé, 1986, «Langage non-verbal : une autre dimension de la communication africaine ». Éthiopiques : revue négro-africaine de littérature, 37-38: 105-117.

Fortin, Sylvie, Marie Nathalie Le Blanc et Josianne Le Gall, 2008, «Entre la oumma, l'ethnicité et la culture : le rapport à l'islam chez les musulmans francophones de Montréal », Diversité urbaine, 18(2): 99-134.

Frazer, James G., 1900, The Golden Bough. A Study in Magic and Religion, London : MacMillan.

Hamès, Constant, 2007, Coran et talismans. Textes et pratiques magiques en milieu musulman. Paris : Karthala Editions.

Hubert, Henri, et Marcel Mauss, 1968, Les fonctions sociales du sacré. Paris, Minuit.

Khan, Naveeda, 2006, «Of children and jinn : an inquiry into an unexpected friendship during uncertain times », Cultural Anthropology, 21(2): 234-264.

Irby, Jon P., 1993, « The Islamic Dialogue with African Traditional Religion. Divination and Health Care », Social Science and Medicine, 36(3): 237-247.

Islam, F. et R.A. Campbell, 2014, « "Satan Has Afflicted Me!” Jinn- 
Possession and Mental Illness in the Qur'an », Journal of religion and health, 53(1) : 229-243.

Lazreg, Marnia (dir.), 1994, The eloquence of silence: Algerian women in question. East Sussex, UK: Psychology Press.

LeBlanc, Marie Nathalie, 2002, « Processes of identification among Frenchspeaking West African migrants in Montreal ». Canadian Ethnic Studies Journal, 34(3) : 121-141.

Lévi-Strauss, Claude, 1962, La pensée sauvage. Paris : Plon.

Levtzion, Nehemia, 1979, «Patterns of Islamization in West Africa ». Dans

Nehemia Levtzion (dir.), Conversion to Islam : 207-216. New York : Holmes \& Meier.

Mamdani, Mahmood, 2002, "Good Muslim, bad Muslim: a political perspective on culture and terrorism », American Anthropologist, 104 : 766-775.

Ministère de l'immigration et des communautés culturelles du Québec, 2008, Portraits régionaux 1997-2006, Caractéristiques des immigrants établis au Québec et dans les régions en 2008. Québec : Gouvernement du Québec.

Monteil, Vincent, 1964, L'Islam noir. Paris : Éditions du Seuil.

Moreau, René Luc, 1982, Africains musulmans. Paris : Présence Africaine; Abidjan : INADES Éditions.

Odugbesan, Clara, 1969, "Femininity in Yoruba Religious Art ». Dans Mary Douglas et Phyllis Mary Kaberry (dir.), Man in Africa: 201-213. London : Oxford University Press.

Peek, Philip M., 1991, «Introduction ». Dans Philip Peek (dir.), African divination systems. Ways of knowing : 1-22. Georgetown: Georgetown University Press.

Regourd, Anne, 2000, « Le jet de coquillages divinatoire en islam arabe et en Afrique subsaharienne: première contribution à une étude comparative ». Journal of Oriental and African Studies 11 : 133-149.

Rothenberg, Celia, 2006, "My Wife is from the Jinn. Palestinian Men, Diaspora and Love ». Dans Lahoucine Ouzgane (dir.), Islamic Masculinities : 89-104. London: Zed Books.

Shaw, Rosalind, 1992, "Dreaming as Accomplishment : Power, the Individual, and Temne divination ». Dans Rosalind Shaw et M. Charles Jedrej, (dir.) Dreaming, religion, and society in Africa : 35-54. Leiden : Brill.

Soares, Benjamin, 2005, Islam and the Prayer Economy: History and Authority in a Malian Town. Ann Arbor : University of Michigan Press.

Sow, Ibrahima, 2006, « Le Listixaar est-il une pratique divinatoire? » 
Ethiopiques, 77 : 193-206.

Sow, Ibrahima, 2004, «Les représentations sénégalaises du destin ». Notes Africaines, 207-208: http://bca.ucad.sn/jspui/handle/01/135 [récupéré le 16 juillet 2010].

Sow, Ibrahima, 1977, Psychiatrie dynamique africaine. Paris : Payot.

Tall, Cheikh Ahmadou, 1996, Les Dimensions de l'islam (5e éd.). Beyrouth : Dar el Fikr.

Triaud, Jean-Louis, 1974, «Un cas de passage collectif à l'islam en Basse Côte d'Ivoire : le village d'Ahua au début du siècle ", Cahiers d'études africaines $14(54)$ : 317-337.

Werbner, Pnina, 2015, « Sacrifice, purification and gender In the Hajj: personhood, metonymy, and ritual transformation ». Dans Luitgard Mois et Marjo Buitelaar (dir.), Hajj: Global Interactions through Pilgrimage : 27-40. Leiden : Sidestone Press. 